\title{
Type 2 diabetes mellitus, brain atrophy and cognitive decline in older people: a longitudinal study
}

\author{
Michele L. Callisaya ${ }^{1,2,3} \cdot$ Richard Beare ${ }^{2,4} \cdot$ Chris Moran $^{2,5,6} \cdot$ Thanh Phan $^{3} \cdot$ Wei Wang ${ }^{2}$ - Velandai K. Srikanth ${ }^{1,2,5}$
}

Received: 17 May 2018 / Accepted: 22 October 2018 / Published online: 13 December 2018

(C) Springer-Verlag GmbH Germany, part of Springer Nature 2018

\begin{abstract}
Aims/hypothesis The aims of the study were to examine whether type 2 diabetes mellitus is associated with greater brain atrophy and cognitive decline, and whether brain atrophy mediates associations between type 2 diabetes and cognitive decline.

Methods Participants without dementia aged 55-90 years from the Cognition and Diabetes in Older Tasmanians (CDOT) study underwent brain MRI (ventricular and total brain volume) and neuropsychological measures (global function and seven cognitive domains) at three time points over 4.6 years. Mixed models were used to examine longitudinal associations of type 2 diabetes with cognitive and MRI measures, adjusting for covariates. A test of mediation was used to determine whether brain atrophy explained associations between type 2 diabetes and cognitive decline.

Results A total of 705 participants (diabetes: $n=348$, mean age 68.2 years [SD 7.0]; no diabetes: $n=357$, mean age 72.5 years [SD 7.1]) were available at baseline. Adjusting for age, sex, education and vascular risk factors, there were significant diabetes $\times$ time interactions for verbal memory $(\beta-0.06 ; 95 \% \mathrm{CI}-0.09,-0.02)$ and verbal fluency $(\beta-0.03 ; 95 \% \mathrm{CI}-0.06,-0.00)$. Although people with diabetes had lower brain $(\beta-14.273 ; 95 \% \mathrm{CI}-21.197,-6.580)$ and greater ventricular $(\beta 2.672 ; 95 \% \mathrm{CI}$ $0.152,5.193)$ volumes at baseline, there were no significant diabetes $\times$ time interactions $(p>0.05)$ or evidence of mediation of the diabetes-cognition relationship by brain atrophy.

Conclusions/interpretation In older community-dwelling people, type 2 diabetes is associated with decline in verbal memory and fluency over $\sim 5$ years. The effect of diabetes on brain atrophy may begin earlier (midlife).
\end{abstract}

Keywords Brain atrophy $\cdot$ Brain imaging $\cdot$ Cognition $\cdot$ Dementia $\cdot$ Longitudinal study $\cdot$ Type 2 diabetes mellitus

$\overline{\text { Electronic supplementary material The online version of this article }}$ (https://doi.org/10.1007/s00125-018-4778-9) contains peer-reviewed but unedited supplementary material, which is available to authorised users.

Michele L. Callisaya

michele.callisaya@utas.edu.au

1 Menzies Institute for Medical Research, University of Tasmania, 17 Liverpool Street, Private Bag 23, Hobart, TAS 7000, Australia

2 Peninsula Clinical School, Central Clinical School, Monash University, Melbourne, VIC, Australia

3 Stroke and Aging Research Group, Department of Medicine, School of Clinical Sciences, Monash University, Melbourne, VIC, Australia

4 Developmental Imaging, Murdoch Children's Research Institute, Melbourne, VIC, Australia

5 Department of Geriatric Medicine, Frankston Hospital, Peninsula Health, Melbourne, VIC, Australia

6 Department of Aged Care, Alfred Health, Melbourne, VIC, Australia

\section{Abbreviations \\ DBP Diastolic blood pressure \\ SBP Systolic blood pressure}

WMH White matter hyperintensity

\section{Introduction}

Dementia is a major public health problem [1]. The burden of poor metabolic health is also rising worldwide and is a strong risk factor for dementia [1]. The presence of type 2 diabetes mellitus, in particular, doubles the risk of dementia in older age [2]. Findings from the majority of longitudinal studies suggest that type 2 diabetes is associated with a greater decline in executive function [3-5], processing speed [4, 6-8], verbal fluency [6] and memory $[3,4,6]$, while the results of a few studies suggest no associations in these domains $[3,5,9]$. 


\section{Research in context}

\section{What is already known about this subject?}

- Very few studies, to our knowledge, have examined associations between type 2 diabetes and brain atrophy

- None have measured the impact of type 2 diabetes on both brain atrophy and cognitive decline in the same study

What is the key question?

- Is type 2 diabetes associated with brain atrophy, and does atrophy mediate the association between type 2 diabetes and cognitive decline?

What are the new findings?

- Although brain volume was lower at baseline in people with type 2 diabetes, it did not decline at a greater rate over time, or mediate the association between type 2 diabetes and cognitive decline

How might this impact on clinical practice in the foreseeable future?

- The effect of type 2 diabetes on brain atrophy may begin earlier than old age (for example, in midlife). Alternatively, its impact on brain atrophy and cognitive decline in old age may be amplified by the development of substantially greater cerebrovascular pathology (although, this was not observed in our sample, which had fairly low levels of cerebrovascular pathology). Further study of these issues may clarify when and how to intervene to reduce the impact of type 2 diabetes on the brain

There is great interest in identifying pathways linking diabetes and cognitive decline. Type 2 diabetes is associated with lower total brain volume [10], more infarcts [11, 12] and greater white matter hyperintensity (WMH) volume [11, 12]. Cross-sectional analyses suggest that lower grey matter volume $[11,13]$ may substantially mediate the association between type 2 diabetes and cognitive function. However, in the Action to Control Cardiovascular Risk in Diabetes (ACCORD)-Memory in Diabetes (MIND) trial, the less intensive blood pressure and more intensive glycaemic control arms resulted in greater preservation of brain volume but not cognition $[14,15]$. In the handful of longitudinal analyses on this topic, type 2 diabetes has been linked to an increase in ventricular volume [16-18]; a greater decline in total brain volume has been reported in some [19] but not all studies $[17,18,20]$. To our knowledge, there have been no longitudinal studies exploring whether brain atrophy mediates the association between type 2 diabetes and cognitive decline.

In a sample of community-dwelling older people without a history of dementia, we hypothesised that: (1) people with type 2 diabetes would have a greater rate of decline in cognition and total brain volume, and a greater increase in ventricular volume, compared with non-diabetic individuals; (2) these measures of brain atrophy would mediate associations between type 2 diabetes and cognitive decline.

\section{Methods}

Study population Participants aged $\geq 55$ years were recruited from the National Diabetes Service Scheme register in Southern Tasmania (postcodes 7000-7199) into the Cognition and Diabetes in Older Tasmanians (CDOT) longitudinal study between January 2008 and January 2010. The study was designed to examine the effect of type 2 diabetes on cognition and its underlying brain pathways. The National Diabetes Service Scheme register is run by Diabetes Australia and provides support to people with type 2 diabetes. Diagnosis is made by a physician using standard criteria (fasting plasma glucose $\geq 7.0 \mathrm{mmol} / 1$, random plasma glucose $\geq 11.1 \mathrm{mmol} / 1$ or $2 \mathrm{~h}$ plasma glucose $\geq 11.1 \mathrm{mmol} / 1$ after an oral glucose tolerance test). Participants without type 2 diabetes were recruited from the longitudinal population-based Tasmanian Study of Cognition and Gait (TASCOG) and randomly selected using the Southern Tasmanian electoral roll from the same postcodes between December 2004 and 2010. In both studies participants were contacted by letter asking them to participate; they then attended an appointment at the University of Tasmania. Exclusion criteria were residence in a nursing home, insufficient English for cognitive testing, any contraindication to MRI scan, and a history of dementia or Parkinson's disease (determined by a standardised questionnaire). Incident type 2 diabetes at follow-up was defined as diagnosis by a physician, $\mathrm{HbA}_{1 \mathrm{c}}$ $\geq 48 \mathrm{mmol} / \mathrm{mol}(6.5 \%)$ or fasting plasma glucose $\geq 7.0 \mathrm{mmol} / \mathrm{l}$.

Ethics approval was obtained from the Southern Tasmanian Health and Medical Human Research Ethics Committee and the Monash University Human Research Ethics Committee. Informed written consent was obtained from all participants.

Measurements Both groups were followed up approximately 2 and 4 years after the baseline assessment. Measurement of all variables (including fasting blood tests) was identical in both cohorts. 
Cognitive tests and derivation of cognitive scores A comprehensive battery of neuropsychological tests was used to measure: (1) verbal fluency, using the Controlled Oral Word Association Test (with the letters F, A and S; category fluency, animals) [21]; (2) executive function interference, using the Victoria Stroop Test (and the colour minus word subtests) [22]; (3) working memory, using the digit span subtest of the Wechsler Adult Intelligence Scale-Third Edition [23]; (4) attention-processing speed, using the Victoria Stroop dot tests, symbol search and digit symbol-coding subtests of the Wechsler Adult Intelligence Scale-Third Edition [23]; (5) visuospatial ability, using the Rey-Osterrieth Complex Figure Test, copy task [21]; (6) verbal memory, using the Hopkins Verbal Learning Test-Revised generating scores for total immediate recall, delayed recall and recognition memory [21]; (7) visual memory, with a delayed reproduction after 20 min of the Rey-Osterrieth Complex Figure Test [21]. For individual tests, scores were standardised at each visit by creating $z$ scores using the mean and SD from the baseline visit. These domain scores were averaged to create a global cognitive score and also average scores for each of the seven listed cognitive domains. Domain scores with more than one cognitive test were restandardised to an SD of 1 . These scores were used in the regression analysis to allow comparison of associations across cognitive domains, as has been done previously $[3,6,9]$.

MRI brain (total brain and lateral ventricular volume) Brain MRI prior to January 2011 was performed using a 1.5 Tesla scanner (LX Horizon; General Electric, Milwaukee, WI, USA) with the following sequences: high-resolution T1weighted spoiled gradient echo (repetition time [TR] $35 \mathrm{~ms}$, echo time [TE] $7 \mathrm{~ms}$, flip angle $35^{\circ}$, field of view $240 \times$ $240 \mathrm{~mm}$, voxel size $1 \mathrm{~mm}^{3}$ ) comprising 120 contiguous slices; fluid-attenuated inversion recovery (FLAIR) (TR $8802 \mathrm{~ms}$, TE $130 \mathrm{~ms}$, inversion time $2200 \mathrm{~ms}$, voxel size $0.50 \times$ $0.50 \times 3 \mathrm{~mm})$. MRI after January 2011 was performed using a new 1.5 Tesla scanner (Syngo; Siemens, Erlangen, Germany) with the following sequences: high-resolution T1weighted magnetisation-prepared gradient echo (MPRAGE) (TR $1910 \mathrm{~ms}$, TE $3.14 \mathrm{~ms}$, flip angle $15^{\circ}$, field of view $235 \times$ $250 \mathrm{~mm}$, voxel size $1 \mathrm{~mm}^{3}$ ) comprising 160 contiguous slices; FLAIR (TR $8500 \mathrm{~ms}$, TE $92 \mathrm{~ms}$, inversion time $2438 \mathrm{~ms}$, voxel size $0.9 \times 0.9 \times 3.5 \mathrm{~mm}$ ). Eleven participants were scanned on the new and old scanner and calibration factors were computed using linear regression, such that rates of change of volume between the previous time point and each calibration scan were matched. T1-weighted and FLAIR scans for each participant were aligned using the co-registration facility of SPM12 (www.fil.ion.ucl.ac.uk/spm). The FreeSurfer version 5.3 longitudinal pipeline [24] was used to estimate total brain volume, lateral ventricular volume and total intracranial volume with correction of intermediate stages of
FreeSurfer segmentation for WMH masks created from FLAIR scans. WMH volume at baseline was computed using established segmentation procedures; MRI infarcts or cerebral microbleeds at baseline were determined by consensus between expert raters [25]. All image analyses were blinded to age, sex and cognitive outcome measures.

Covariates Potential covariates included baseline age (centred to 55 years), sex, education (years) and self-reported vascular risk history of previous or current smoking (yes/no), myocardial infarct (yes/no), stroke (yes/no), hypertension (self-report, taking antihypertensive medication or having a systolic blood pressure $[\mathrm{SBP}] \geq 140 \mathrm{mmHg}$ or a diastolic blood pressure [DBP] $\geq 90 \mathrm{mmHg}$ ), hypercholesterolaemia (yes/no), BMI, depression (Geriatric Depression Scale) and ApoE4 (also known as $A P O E$ ) genotype (whole blood DNA).

Statistics Non-normal variables were transformed as required. Differences between diabetes status and baseline characteristics were examined using $t$ tests and $\chi^{2}$ tests. For longitudinal analyses, mixed models (maximum likelihood estimation, unstructured covariance) were used to examine the associations between baseline diabetes status and change in MRI brain measures, global cognitive function and the individual cognitive domains. Fixed effects were terms for time since baseline and main effects were for diabetes status and an interaction between diabetes and time. Random effects for the intercept and slope were fitted for each individual, allowing participants to have different scores at baseline and rates of change in the dependent variable (MRI brain or cognitive measures). Model 1 was adjusted for baseline age, sex and education (and total intracranial volume for MRI measures). Model 2 included the covariates at baseline and their interaction with time in order to adjust for their effect on the relationship between type 2 diabetes and MRI brain/cognition over time. Covariates in model 2 were included if they changed the coefficient of the diabetes $\times$ time interaction by more than $10 \%$. Three-way interactions were also explored between type 2 diabetes, time and the following covariates: age (greater than or less than 65 years of age), sex and ApoE4 status. For MRI outcome variables, additional three-way interactions were explored for baseline WMH volume, infarcts and microbleeds.

To examine whether MRI markers of atrophy mediated any associations between type 2 diabetes and decline in cognition, we entered the MRI variables and their interaction with time into models of type 2 diabetes and cognitive decline. As in our prior study, if the MRI measure attenuated the $\beta$ coefficient for diabetes $\times$ time (by $>30 \%$ ), and the coefficient of the MRI measure remained unchanged from its unadjusted value without diabetes in the model, it was considered a potential mediator [11].

We performed three sensitivity analyses. First, we used multiple imputation to replace missing data during follow-up and repeated our analyses. Multiple imputation is likely to 
improve precision (reduce bias) when compared with more traditional approaches for dealing with missing data [26]. It is based on the assumption that the missing data are missing at random. Although missing at random is empirically unverifiable [27], individuals with cognitive impairment and other conditions at baseline were more likely to drop out of the study than were individuals without health conditions, suggesting that the probability of dropout depended on observed baseline measures but not the unobserved outcomes. Unrestricted model-based multiple imputation using Bayesian estimation [28] was used to impute the missing values because such a model is general enough so that model misspecification cannot occur [29]. The baseline measures (age, sex, education, type 2 diabetes status, ApoE4 genotype, vascular risk factors, cognitive measures and all MRI brain variables) were included, reducing the uncertainty caused by the missing values and therefore improving the precision of the estimation [30]. Multiple imputation involved taking five copies of the dataset [31] and imputing the missing values in each copy. The model was fitted separately to each of the five complete datasets, and the inference was carried out by combining the estimates and standard errors of each variable of interest across the five completed datasets using Rubin's rules [32]. The resulting multiple imputation estimates are the average of the variable estimates across the five imputed datasets. We also performed 'worst and best case' scenarios by giving any missing outcome a value of 2 SDs below or above the sample mean. Analysis was performed using STATA version 15 (StataCorp, College Station, TX, USA).

\section{Results}

There were 705 participants in the study at phase 1 (diabetes, $n=348$; no diabetes, $n=357$ ). Phase 2 mean follow-up time was 2.6 years (SD 0.44, median 2.6, interquartile range 2.32.8 ) and phase 3 mean follow-up time was 4.6 years (SD 0.53, median 4.4, interquartile range 4.2-5.0). Five participants had missing cognitive data but did have MRI scans. There were 506 participants at phase 2 (two with missing cognitive data but with MRI scans) and 431 at phase 3 (one with missing cognitive data but with an MRI scan). MRI scans were performed in participants with no contraindications (claustrophobia or metal implants). The total number of MRI scans was as follows: phase $1, n=602$; phase 2, $n=385$; phase 3, $n=295$. Overall, those lost to follow-up were older $(p<0.001)$, had higher $\mathrm{HbA}_{1 \mathrm{c}}(p=0.01)$, were more likely to have had a myocardial infarct $(p=0.001)$ or stroke $(p=0.001)$, and had lower baseline total global cognition $z$ score $(p=0.002)$, higher ventricular volume $(p=0.008)$ and lower brain volume $(p=$ $0.001)$. There was no difference in dropout by type 2 diabetes status $(p=0.47)$, sex $(p=0.16)$, education $(p=0.31)$, hypertension $(p=0.94)$, high cholesterol $(p=0.33)$ or BMI $(p=$
0.85). Seven people developed incident type 2 diabetes during follow-up.

Participant characteristics by diabetes status are provided in Table 1 . Those with type 2 diabetes were more likely to be younger $(p<0.001)$, on blood pressure- and lipid-lowering medication $(p<0.001)$, and have a history of high blood pressure and high cholesterol $(p<0.001)$. Those with type 2 diabetes also had a higher BMI $(p<0.001)$ and higher depression scores $(p=0.002)$. SBP $(p=0.001)$ and DBP $(p<0.001)$ were slightly higher in those without type 2 diabetes. The mean duration of diabetes was 9.5 years (SD 9.4); 71 participants were on insulin therapy.

Type 2 diabetes and cognitive decline At baseline, type 2 diabetes was associated with lower attention-processing speed $(\beta-0.19 ; 95 \% \mathrm{CI}-0.36,-0.02 ; p=0.03)$, visuospatial ability $(\beta-0.68 ; 95 \% \mathrm{CI}-0.84,-0.53 ; p<0.001)$ and visual memory $(\beta-0.54 ; 95 \% \mathrm{CI}-0.69,-0.38 ; p<0.001)$, but not other cognitive variables $(p>0.05)$. Table 2 (model 1$)$ shows the age-, sex- and education-adjusted associations between type 2 diabetes at baseline and cognitive decline over time. Type 2 diabetes was associated with a greater decline in the domains of verbal fluency ( $\beta-0.04 ; 95 \% \mathrm{CI}-0.07,-0.02 ; p=0.001)$ and verbal memory $(\beta-0.05 ; 95 \% \mathrm{CI}-0.08,-0.02 ; p=$ $0.002)$, and with a trend for working memory $(\beta-0.02$; $95 \% \mathrm{CI}-0.05,-0.00 ; p=0.05)$. Although visuospatial function and visual memory were lower in people with type 2 diabetes at baseline and subsequent assessments, they declined at a slower rate in people with type 2 diabetes (visuospatial function: $\beta 0.14 ; 95 \%$ CI $0.11,0.18 ; p<0.001$; visual memory: $\beta 0.10 ; 95 \%$ CI $0.07,0.13 ; p<0.001)$. Type 2 diabetes was not associated with a greater rate of decline in global cognitive score $(\beta-0.03 ; 95 \% \mathrm{CI}-0.07,0.01 ; p=0.17)$, attention-processing speed $(\beta-0.01 ; 95 \% \mathrm{CI}-0.23,0.01$; $p=0.37)$ or executive function interference score $(\beta-0.03$; $95 \%$ CI $-0.08,0.02 ; p=0.20$ ).

In fully adjusted models (Table 2 , model 2 ) the association between type 2 diabetes and greater decline in verbal fluency ( $\beta-0.03$; 95\% CI $-0.06,-0.00)$ (Fig. 1$)$ and verbal memory $(\beta-0.06$; 95\% CI $-0.09,-0.02$ ) (Fig. 1$)$, and between type 2 diabetes and slower decline in visuospatial ability $(\beta 0.14$; $95 \%$ CI $0.11,0.18)$ and visual memory $(\beta 0.11 ; 95 \% \mathrm{CI}$ $0.08,0.14)$ remained significant. The association with working memory was no longer significant $(\beta-0.02 ; 95 \% \mathrm{CI}$ $-0.05,0.01)$. Three-way interaction terms of diabetes and time did not suggest increased rates of decline in people of older age, ApoE4 carriers or either sex $(p>0.05)$. Results in all models did not change meaningfully when the seven participants with incident diabetes during follow-up were removed. Table 3 shows the associations after imputing missing data. There was little change in the strength of the majority of associations, except for verbal fluency, which weakened $(\beta$ $-0.02 ; 95 \%$ CI $-0.06,0.01)$. Table 4 shows the results of the 
Table 1 Participant characteristics $(n=705)$

\begin{tabular}{|c|c|c|}
\hline Characteristic & $\begin{array}{l}\text { No type } 2 \\
\text { diabetes } \\
(n=357)\end{array}$ & $\begin{array}{l}\text { Type } 2 \\
\text { diabetes } \\
(n=348)\end{array}$ \\
\hline Age, years & $72.5 \pm 7.1$ & $68.2 \pm 7.0$ \\
\hline Female & $167(46.8)$ & $139(39.9)$ \\
\hline \multicolumn{3}{|l|}{ Race } \\
\hline European descent & $354(99.2)$ & $343(98.6)$ \\
\hline Asian & $2(0.6)$ & $5(1.4)$ \\
\hline Mixed (African/Asian) & $1(0.3)$ & $0(0.0)$ \\
\hline Education & $10.8 \pm 3.7$ & $11.2 \pm 3.5$ \\
\hline Hypertension $^{\mathrm{a}}$ & $269(75.4)$ & $312(89.7)$ \\
\hline Angina & $41(11.5)$ & $60(17.2)$ \\
\hline Myocardial infarct & $44(12.3)$ & $53(15.2)$ \\
\hline Stroke & $24(6.7)$ & $36(10.3)$ \\
\hline High cholesterol & $137(38.4)$ & $255(73.3)$ \\
\hline Insulin therapy & & $71(20.4)$ \\
\hline Short acting & & $8(2.3)$ \\
\hline Intermediate acting & & $43(12.4)$ \\
\hline Long acting & & $20(5.7)$ \\
\hline $\mathrm{HbA}_{1 \mathrm{c}}, \mathrm{mmol} / \mathrm{mol}$ & 38 & 55 \\
\hline $\mathrm{HbA}_{1 \mathrm{c}}, \%$ & $5.6 \pm 0.3$ & $7.2 \pm 1.2$ \\
\hline Fasting glucose, $\mathrm{mmol} / \mathrm{l}$ & $5.3 \pm 0.5$ & $7.7 \pm 2.3$ \\
\hline Type 2 diabetes duration, years & & $9.5 \pm 9.4$ \\
\hline Past or present smoker & $179(50.1)$ & $190(54.6)$ \\
\hline ApoE4 & $82(23.0)$ & $80(23.0)$ \\
\hline BMI, kg/m² & $27.4 \pm 4.3$ & $30.7 \pm 5.4$ \\
\hline Geriatric Depression Scale score & $1.9 \pm 2.2$ & $2.5 \pm 2.65$ \\
\hline Blood pressure-lowering medication & $177(49.6)$ & $258(74.1)$ \\
\hline Lipid-lowering medication & $98(27.5)$ & $235(67.5)$ \\
\hline Glucose-lowering medication & & $234(67.2)$ \\
\hline \multicolumn{3}{|l|}{ Cognitive and brain variables } \\
\hline Hopkins immediate recall & $22.1 \pm 6.2$ & $23.6 \pm 5.5$ \\
\hline Hopkins delayed recall & $7.6 \pm 3.0$ & $8.0 \pm 2.8$ \\
\hline Hopkins recognition memory & $9.9 \pm 2.0$ & $10.1 \pm 1.7$ \\
\hline Rey Complex Figure Test, copy task & $32.0 \pm 5.1$ & $28.2 \pm 6.2$ \\
\hline $\begin{array}{l}\text { Rey Complex Figure Test, delayed } \\
\text { recall }\end{array}$ & $14.9 \pm 6.9$ & $12.9 \pm 6.5$ \\
\hline Digit symbol coding & $50.4 \pm 15.2$ & $52.3 \pm 14.1$ \\
\hline Symbol search & $22.7 \pm 7.7$ & $24.4 \pm 7.5$ \\
\hline Digit span & $15.8 \pm 3.9$ & $16.3 \pm 3.6$ \\
\hline COWAT category & $17.2 \pm 4.8$ & $18.4 \pm 4.8$ \\
\hline COWAT word & $36.5 \pm 12.9$ & $35.8 \pm 12.7$ \\
\hline Stroop dot time & $15.5 \pm 4.4$ & $16.0 \pm 5.0$ \\
\hline Stroop dot word & $21.3 \pm 8.0$ & $20.1 \pm 5.9$ \\
\hline Stroop dot colour & $38.1 \pm 21.9$ & $36.3 \pm 14.8$ \\
\hline Total brain volume, $\mathrm{ml}$ & $897.55 \pm 91.05$ & $908.91 \pm 97.32$ \\
\hline Lateral ventricular volume, $\mathrm{ml}$ & $30.45 \pm 17.36$ & $29.92 \pm 15.65$ \\
\hline $\mathrm{WMH}, \mathrm{ml}$ & $4.70 \pm 7.10$ & $3.91 \pm 5.96$ \\
\hline Infarcts & $53(17.0)$ & $66(22.7)$ \\
\hline Microbleeds & $23(7.4)$ & $12(4.1)$ \\
\hline
\end{tabular}

Data are $n(\%)$ or mean \pm SD; MRI variables: no type 2 diabetes $(n=311)$ and type 2 diabetes $(n=291)$

${ }^{a}$ Hypertension was defined as a history of high blood pressure, taking blood pressure-lowering medications, SBP $>140 \mathrm{mmHg}$ or DBP $>90 \mathrm{mmHg}$

COWAT, Controlled Oral Word Association Test

worst-case scenario, where the association between type 2 diabetes and verbal fluency weakened slightly $(\beta-0.01$; $95 \%$ CI $-0.05,0.03)$. Electronic Supplementary Material (ESM) Table 1 shows the more unlikely best-case scenario, where the association between type 2 diabetes and verbal fluency was of similar strength but non-significant $(\beta-0.03$; $95 \% \mathrm{CI}-0.08,0.02$ ).

Type 2 diabetes and brain atrophy At baseline, type 2 diabetes was associated with lower total brain volume $(\beta-14.273$; $95 \%$ CI $-21.197,-6.580 ; p<0.001)$ and higher ventricular volume ( $\beta 2.672 ; 95 \%$ CI $0.152,5.193 ; p=0.04)$. Table 2 shows the associations between type 2 diabetes at baseline and MRI measures over time: model 1 is adjusted for baseline age, sex and education (as for the analysis of cognition), as well as total intracranial volume; model 2 is adjusted for the same covariates as for the analysis of cognition. Total brain volume decreased $(\beta-8.481 ; 95 \% \mathrm{CI}-9.863,-7.099)$ and lateral ventricular volume increased $(\beta 1.142 ; 95 \%$ CI 0.954 , 1.331 ) over time in the overall sample. There were no statistically significant interactions between diabetes and time in explaining changes in total brain volume $(\beta-0.451 ; 95 \% \mathrm{CI}$ $-1.807,0.905 ; p=0.51)$ and ventricular volume $(\beta 0.175$; $95 \% \mathrm{CI}-0.009,0.359 ; p=0.06)$. Three-way interaction terms of diabetes and time were not statistically significant with age, sex, ApoE4, baseline WMH volume, infarcts or microbleeds $(p>0.05)$. There were also no statistically significant associations when data were imputed (Table 3), in a worst case (Table 4) or best case (ESM Table 1) scenario.

Type 2 diabetes, brain atrophy and cognition The addition of total brain or lateral ventricular volume did not alter the associations between type 2 diabetes and decline in verbal memory or fluency (results not shown).

\section{Discussion}

In older community-dwelling people without a history of dementia, type 2 diabetes was associated with reduced cognitive function at baseline and a greater decline in verbal memory and verbal fluency independently of confounding factors. Although type 2 diabetes was associated with lower total brain volume and higher ventricular volume at baseline, it was not associated with the rate of brain atrophy and atrophy did not mediate associations between type 2 diabetes and cognitive decline.

Type 2 diabetes and cognitive decline We confirmed that type 2 diabetes was associated with poorer baseline cognitive function, suggesting an impact on cognitive reserve that may begin before older age. In addition, even over a relatively short period of $\sim 5$ years, we found that type 2 diabetes was associated with faster decline in verbal fluency (a measure of executive ability) and memory. For example, in people without type 2 diabetes, verbal fluency slightly increased on average each year $(0.004 \mathrm{SD} /$ units per year), whereas it declined at more than triple the rate in those with type 2 diabetes $(-0.023 \mathrm{SD} /$ 
Table 2 Difference in change over time between no diabetes and type 2 diabetes

\begin{tabular}{|c|c|c|c|c|c|c|}
\hline \multirow[t]{2}{*}{ Variable } & \multicolumn{3}{|l|}{ Model 1} & \multicolumn{3}{|l|}{ Model 2} \\
\hline & $\beta$ coefficient & $95 \% \mathrm{CI}$ & $p$ value & $\beta$ coefficient & $95 \% \mathrm{CI}$ & $p$ value \\
\hline \multicolumn{7}{|l|}{ Cognitive $z$ scores } \\
\hline \multicolumn{7}{|l|}{ Total global cognition } \\
\hline Time & -0.01 & $-0.04,0.01$ & & -0.02 & $-0.07,0.03$ & \\
\hline Type 2 diabetes & -0.14 & $-0.28,-0.00$ & & -0.05 & $-0.21,0.10$ & \\
\hline Type 2 diabetes $\times$ time & -0.03 & $-0.07,0.01$ & 0.17 & -0.03 & $-0.07,0.01$ & 0.18 \\
\hline \multicolumn{7}{|l|}{ Attention-processing speed } \\
\hline Time & -0.02 & $-0.03,-0.01$ & & -0.00 & $-0.03,0.02$ & \\
\hline Type 2 diabetes & -0.17 & $-0.30,-0.04$ & & -0.13 & $-0.27,0.01$ & \\
\hline Type 2 diabetes $\times$ time & -0.01 & $-0.23,0.01$ & 0.37 & -0.00 & $-0.02,0.02$ & 0.93 \\
\hline \multicolumn{7}{|l|}{ Verbal fluency } \\
\hline Time & 0.01 & $-0.00,0.03$ & & 0.04 & $0.01,0.08$ & \\
\hline Type 2 diabetes & -0.05 & $-0.19,0.09$ & & -0.06 & $-0.21,0.10$ & \\
\hline Type 2 diabetes $\times$ time & -0.04 & $-0.07,-0.02$ & 0.001 & -0.03 & $-0.06,-0.00$ & 0.05 \\
\hline \multicolumn{7}{|l|}{ Executive function } \\
\hline Time & 0.01 & $-0.03,0.04$ & & -0.01 & $-0.07,0.05$ & \\
\hline Type 2 diabetes & 0.02 & $-0.13,0.16$ & & 0.11 & $-0.05,0.27$ & \\
\hline Type 2 diabetes $\times$ time & -0.03 & $-0.08,0.02$ & 0.20 & -0.04 & $-0.10,0.01$ & 0.15 \\
\hline \multicolumn{7}{|l|}{ Verbal memory } \\
\hline Time & 0.05 & $0.03,0.07$ & & 0.03 & $-0.01,0.07$ & \\
\hline Type 2 diabetes & 0.01 & $-0.12,0.15$ & & 0.03 & $-0.12,0.18$ & \\
\hline Type 2 diabetes $\times$ time & -0.05 & $-0.08,-0.02$ & 0.002 & -0.06 & $-0.09,-0.02$ & 0.001 \\
\hline \multicolumn{7}{|l|}{ Working memory } \\
\hline Time & 0.02 & $-0.00,0.04$ & & 0.04 & $0.01,0.07$ & \\
\hline Type 2 diabetes & 0.01 & $-0.14,0.15$ & & 0.02 & $-0.15,0.18$ & \\
\hline Type 2 diabetes $\times$ time & -0.02 & $-0.05,-0.00$ & 0.05 & -0.02 & $-0.05,0.01$ & 0.15 \\
\hline \multicolumn{7}{|l|}{ Visual memory } \\
\hline Time & -0.16 & $-0.18,-0.14$ & & -0.15 & $-0.19,-0.11$ & \\
\hline Type 2 diabetes & -0.51 & $-0.65,-0.38$ & & -0.54 & $-0.69,-0.39$ & \\
\hline Type 2 diabetes $\times$ time & 0.10 & $0.07,0.13$ & $<0.001$ & 0.11 & $0.08,0.14$ & $<0.001$ \\
\hline \multicolumn{7}{|l|}{ Visuospatial function } \\
\hline Time & -0.29 & $-0.31,-0.26$ & & -0.31 & $-0.35,-0.26$ & \\
\hline Type 2 diabetes & -0.78 & $-0.91,-0.64$ & & -0.72 & $-0.87,-0.57$ & \\
\hline Type 2 diabetes $\times$ time & 0.14 & $0.11,0.18$ & $<0.001$ & 0.14 & $0.11,0.18$ & $<0.001$ \\
\hline \multicolumn{7}{|l|}{ MRI brain measures } \\
\hline \multicolumn{7}{|l|}{ Brain volume } \\
\hline Time & -7.679 & $-8.519,-6.8391$ & & -8.481 & $-9.863,-7.099$ & \\
\hline Type 2 diabetes & -16.969 & $-23.796,-10.143$ & & -14.925 & $-22.515,-7.334$ & \\
\hline Type 2 diabetes $\times$ time & 0.493 & $-0.724,1.709$ & 0.43 & -0.451 & $-1.807,0.905$ & 0.51 \\
\hline \multicolumn{7}{|l|}{ Ventricular volume } \\
\hline Time & 1.187 & $1.074,1.300$ & & 1.142 & $0.954,1.331$ & \\
\hline Type 2 diabetes & 2.291 & $0.057,4.525$ & & 2.484 & $-0.017,4.987$ & \\
\hline Type 2 diabetes $\times$ time & 0.108 & $-0.055,0.272$ & 0.20 & 0.175 & $-0.009,0.359$ & 0.06 \\
\hline
\end{tabular}

All domain $z$ scores renormalised to SD of 1

Model 1 adjusted for baseline age, sex and education, with the addition of total intracranial volume for MRI measures

Model 2 additionally adjusted for baseline stroke, hypertension, high cholesterol and obesity, and their interactions with time 

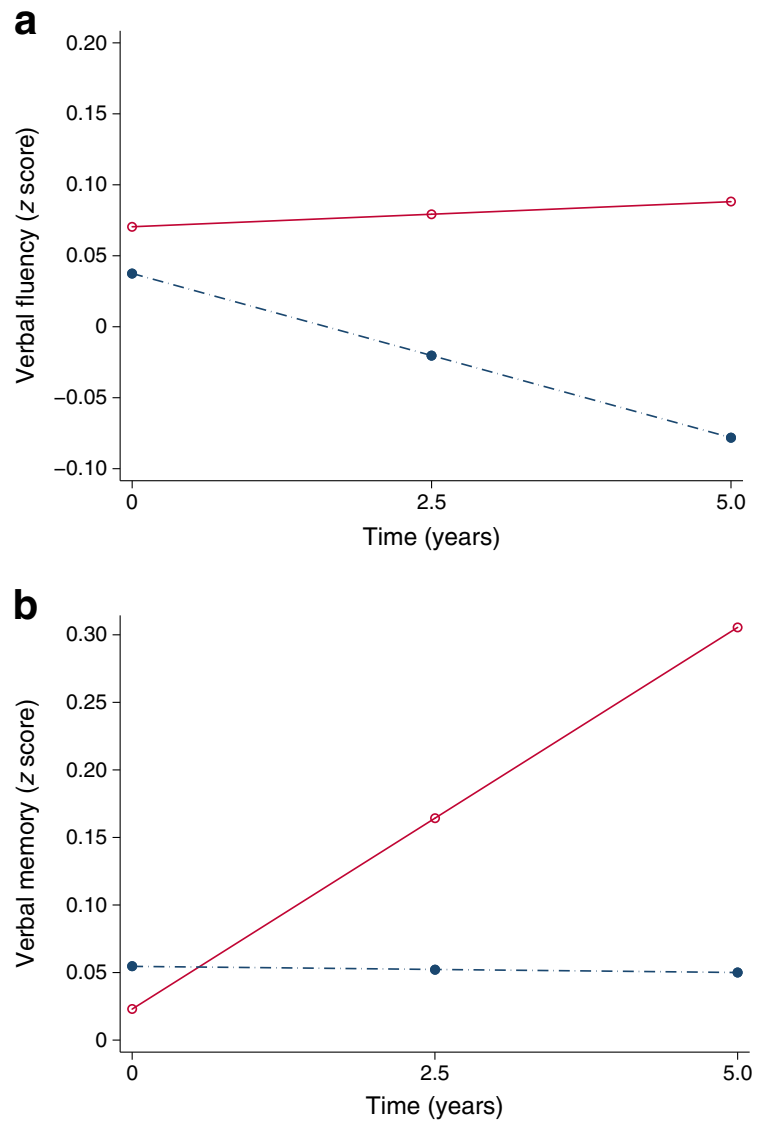

Fig. 1 (a) Longitudinal association between type 2 diabetes and verbal fluency (predictive margins). (b) Longitudinal association between type 2 diabetes and verbal memory (predictive margins). Unbroken line, no type 2 diabetes; broken line, type 2 diabetes

units per year). Such accelerated cognitive decline may contribute to executive difficulties in everyday activities and health behaviours (such as medication compliance), which in turn may poorly influence future vascular health and cognitive decline, and possibly an earlier onset of dementia in those with type 2 diabetes [33]. Previous findings have been mixed, particularly in middle vs older age. Studies commencing in midlife and followed up over 10 years or more tend to show that type 2 diabetes is associated with faster decline in global cognitive function $[3,6]$, processing speed $[4,6]$, executive function $[3,4]$, verbal fluency [6] and memory $[3,4,6]$. However, in older cohorts, these associations are more variable, with some showing greater decline in global cognition [5, 34-36], but not others $[8,9,37]$. The non-significant results in our study for the global cognitive score may be explained by its derivation from a wider range of tests than used in other studies: some of the tests used showed less decline in type 2 diabetes (described further below) and hence a cancelling of effects. Studies in older age have also reported mixed results for specific cognitive domains: some have reported greater decline in verbal fluency [38] and memory [37, 39] in people with type 2 diabetes, whereas several others have reported no differences
$[5,9,34,35,38,40]$. These mixed findings most likely reflect differences in populations, follow-up times and cognitive measures. Surprisingly, we found that visuospatial function and visual memory were slower to decline in people with type 2 diabetes, although those with diabetes performed worse at baseline [11]. A previous study of people with $(n=68)$ and without type 2 diabetes $(n=38)$ also found a similar phenomenon, with healthy people showing a decline in visuospatial function (using a modified version of the Taylor Complex Figure), whereas people with diabetes tended to improve [41].

Type 2 diabetes and brain atrophy Although we found that both ventricular and brain volume were worse at baseline in people with type 2 diabetes, there were no differences in rates of decline. Prior studies are very few and describe mixed results, some reporting type 2 diabetes to be associated with greater rates of increase in ventricular volume [16, 17], but not in decline of total brain volume over time $[17,18,20]$. The difference in baseline volumes in our study suggests that changes in brain atrophy may begin earlier in life, such as in midlife, and carry the potential for reduced brain reserve. Indeed, in a previous study, midlife type 2 diabetes (mean age 54, SD 9.0) was associated with a greater increase in temporal horn volume of the lateral ventricle, thought to be a marker of hippocampal and medial temporal atrophy [18]. The absence of a greater rate of brain atrophy related to diabetes in our sample may be explained partly by the relatively short duration of follow-up, as well as the low burden of cerebrovascular disease [34-36]. In a recent autopsy study of people with a larger burden of cerebrovascular disease than in our sample, the authors reported the combination of diabetes and cerebrovascular disease was associated with lower cognitive scores at the end of life compared with the presence of either one risk factor alone [36]. It is possible that our sample had a lower burden of cerebrovascular disease as a result of relatively good blood glucose and blood pressure control and the high prevalence of blood pressure- and lipidlowering drug use. In the absence of good control of these factors, the greater accrual of cerebrovascular disease may impact on the rates of brain atrophy in diabetes. This theory remains to be tested.

Type 2 diabetes, brain atrophy and cognitive decline Contrary to our hypotheses and results from crosssectional studies $[11,13]$, total brain or lateral ventricular volume did not mediate associations between type 2 diabetes and cognitive decline. In our previous cross-sectional analyses, brain volume appeared to substantially mediate the association between type 2 diabetes and cognition [11] No other studies have compared decline in both cognition and brain atrophy between people with and without type 2 diabetes together in the same study. In the Women's Health Initiative, associations between type 2 diabetes and future 
Table 3 Difference in change over time between no type 2 diabetes and type 2 diabetes with imputed data

\begin{tabular}{|c|c|c|c|}
\hline \multirow[t]{2}{*}{ Variable } & \multicolumn{3}{|c|}{ Model 2 with imputed data } \\
\hline & $\beta$ coefficient & $95 \% \mathrm{CI}$ & $p$ value \\
\hline \multicolumn{4}{|l|}{ Cognitive $z$ scores } \\
\hline \multicolumn{4}{|l|}{ Total global cognition } \\
\hline Time & -0.04 & $-0.60,0.51$ & \\
\hline Type 2 diabetes & 0.07 & $-1.58,1.71$ & \\
\hline Type 2 diabetes $\times$ time & -0.03 & $-0.51,0.46$ & 0.91 \\
\hline \multicolumn{4}{|l|}{ Attention-processing speed } \\
\hline Time & -0.02 & $-0.04,0.00$ & \\
\hline Type 2 diabetes & -0.14 & $-0.28,0.01$ & \\
\hline Type 2 diabetes $\times$ time & 0.00 & $-0.02,0.02$ & 0.67 \\
\hline \multicolumn{4}{|l|}{ Verbal fluency } \\
\hline Time & 0.03 & $-0.01,0.08$ & \\
\hline Type 2 diabetes & -0.06 & $-0.21,0.09$ & \\
\hline Type 2 diabetes $\times$ time & -0.02 & $-0.06,0.01$ & 0.23 \\
\hline \multicolumn{4}{|l|}{ Executive function } \\
\hline Time & -0.01 & $-0.78,0.74$ & \\
\hline Type 2 diabetes & 0.28 & $-1.97,2.54$ & \\
\hline Type 2 diabetes $\times$ time & -0.05 & $-0.71,0.61$ & 0.89 \\
\hline \multicolumn{4}{|l|}{ Verbal memory } \\
\hline Time & 0.02 & $-0.02,0.06$ & \\
\hline Type 2 diabetes & 0.01 & $-0.14,0.16$ & \\
\hline Type 2 diabetes $\times$ time & -0.05 & $-0.08,-0.02$ & 0.002 \\
\hline \multicolumn{4}{|l|}{ Working memory } \\
\hline Time & 0.02 & $-0.01,0.05$ & \\
\hline Type 2 diabetes & 0.03 & $-0.14,0.19$ & \\
\hline Type 2 diabetes $\times$ time & -0.03 & $-0.05,0.00$ & 0.06 \\
\hline \multicolumn{4}{|l|}{ Visual memory } \\
\hline Time & -0.16 & $-0.19,-0.12$ & \\
\hline Type 2 diabetes & -0.53 & $-0.68,-0.39$ & \\
\hline Type 2 diabetes $\times$ time & 0.11 & $0.08,0.14$ & $<0.001$ \\
\hline \multicolumn{4}{|l|}{ Visuospatial function } \\
\hline Time & -0.30 & $-0.34,-0.25$ & \\
\hline Type 2 diabetes & -0.74 & $-0.90,-0.57$ & \\
\hline Type 2 diabetes $\times$ time & 0.15 & $0.10,0.20$ & $<0.001$ \\
\hline \multicolumn{4}{|l|}{ MRI brain measures } \\
\hline \multicolumn{4}{|l|}{ Brain volume } \\
\hline Time & -8.962 & $-10.751,-7.173$ & \\
\hline Type 2 diabetes & -11.565 & $-19.267,-3.864$ & \\
\hline Type 2 diabetes $\times$ time & 0.281 & $-2.116,2.679$ & 0.82 \\
\hline \multicolumn{4}{|l|}{ Ventricular volume } \\
\hline Time & 1.190 & $1.070,1.310$ & \\
\hline Type 2 diabetes & 2.250 & $-0.224,4.724$ & \\
\hline Type 2 diabetes $\times$ time & 0.102 & $-0.068,0.273$ & 0.24 \\
\hline
\end{tabular}

cognitive function (measured only once) were slightly attenuated by adding grey and white matter and ischaemic volumes to the models [20]. In the Prospective Study of Pravastatin in the Elderly at Risk (PROSPER) randomised trial (age 70-82 years; 89 participants with type 2 diabetes and 438 control individuals), baseline lower brain volume was associated with greater decline in an immediate picture-learning task but not in the Stroop Test in people 
Table 4 Difference in change over time between no type 2 diabetes and type 2 diabetes worst case scenario

\begin{tabular}{|c|c|c|c|}
\hline \multirow[t]{2}{*}{ Variable } & \multicolumn{3}{|c|}{ Model 2 with imputed data } \\
\hline & $\beta$ coefficient & $95 \% \mathrm{CI}$ & $p$ value \\
\hline \multicolumn{4}{|l|}{ Cognitive $z$ scores } \\
\hline \multicolumn{4}{|l|}{ Total global cognition } \\
\hline Time & -0.15 & $-0.20,-0.10$ & \\
\hline Type 2 diabetes & -0.05 & $-0.20,0.10$ & \\
\hline Type 2 diabetes $\times$ time & -0.02 & $-0.07,0.02$ & 0.28 \\
\hline \multicolumn{4}{|l|}{ Attention-processing speed } \\
\hline Time & -0.02 & $-0.14,-0.06$ & \\
\hline Type 2 diabetes & -0.01 & $-0.25,0.04$ & \\
\hline Type 2 diabetes $\times$ time & -0.01 & $-0.04,0.03$ & 0.62 \\
\hline \multicolumn{4}{|l|}{ Verbal fluency } \\
\hline Time & -0.08 & $-0.12,-0.04$ & \\
\hline Type 2 diabetes & -0.06 & $-0.22,0.092$ & \\
\hline Type 2 diabetes $\times$ time & -0.01 & $-0.05,0.03$ & 0.52 \\
\hline \multicolumn{4}{|l|}{ Executive function } \\
\hline Time & -0.15 & $-0.21,-0.09$ & \\
\hline Type 2 diabetes & 0.08 & $-0.08,0.24$ & \\
\hline Type 2 diabetes $\times$ time & -0.03 & $-0.09,0.02$ & 0.22 \\
\hline \multicolumn{4}{|l|}{ Verbal memory } \\
\hline Time & -0.08 & $-0.12,-0.03$ & \\
\hline Type 2 diabetes & 0.03 & $-0.11,0.18$ & \\
\hline Type 2 diabetes $\times$ time & -0.05 & $-0.09,-0.01$ & 0.02 \\
\hline \multicolumn{4}{|l|}{ Working memory } \\
\hline Time & -0.09 & $-0.14,-0.04$ & \\
\hline Type 2 diabetes & 0.00 & $-0.16,0.17$ & \\
\hline Type 2 diabetes $\times$ time & -0.02 & $-0.06,0.02$ & 0.29 \\
\hline \multicolumn{4}{|l|}{ Visual memory } \\
\hline Time & -0.25 & $-0.30,-0.21$ & \\
\hline Type 2 diabetes & -0.51 & $-0.66,-0.36$ & \\
\hline Type 2 diabetes $\times$ time & 0.10 & $0.06,0.13$ & $<0.001$ \\
\hline \multicolumn{4}{|l|}{ Visuospatial function } \\
\hline Time & -0.42 & $-0.48,-0.37$ & \\
\hline Type 2 diabetes & -0.69 & $-0.84,-0.53$ & \\
\hline Type 2 diabetes $\times$ time & 0.14 & $0.09,0.19$ & $<0.001$ \\
\hline \multicolumn{4}{|l|}{ MRI brain measures } \\
\hline \multicolumn{4}{|l|}{ Brain volume } \\
\hline Time & -28.487 & $-33.830,-23.163$ & \\
\hline Type 2 diabetes & -16.743 & $-25.742,-7.745$ & \\
\hline Type 2 diabetes $\times$ time & -0.113 & $-4.965,4.739$ & 0.96 \\
\hline \multicolumn{4}{|l|}{ Ventricular volume } \\
\hline Time & -1.850 & $-2.880,-0.820$ & \\
\hline Type 2 diabetes & 2.047 & $-0.537,4.633$ & \\
\hline Type 2 diabetes $\times$ time & 0.091 & $-1.029,0.846$ & 0.84 \\
\hline
\end{tabular}

with type 2 diabetes [19]. Studies which allow for greater accrual of cerebrovascular disease may be more likely to reveal the mediating impact of brain structure on the diabetes-cognitive decline relationship in older age.
Strengths and limitations There are some limitations in our study. It was carried out over a relatively short time frame, and differences between groups in cognition and brain volume may occur over longer periods or earlier in midlife $[3,6]$. 
We had a homogenous sample in reference to race and were therefore unable to explore its effects, whereas prior studies have found stronger associations in African-Americans [5, 38]. As in other longitudinal studies some participants were lost to follow-up, which might have impeded the ability to detect a signal (internal validity). But we used mixed models to enable inclusion of individuals with incomplete follow-up, as well as carrying out three sensitivity analyses to explore its effect. The change in MRI scanners during the middle of the study might have introduced bias in structural brain measures, although we attempted to correct for this using data obtained from the same participant in both scanners. The mean $\mathrm{HbA}_{1 \mathrm{c}}$ for people with type 2 diabetes was relatively low (mean $5.6 \%$ ), which reduces the ability of the study to be generalisable to other populations (external validity). The self-reported method for dementia diagnosis might have influenced findings if there were differences in under-reporting between groups.

Strengths of our study include longitudinal measurement of a wide range of cognitive tests covering different cognitive domains in conjunction with serial brain MRI scans. We explored the independence of associations between type 2 diabetes and cognitive decline for several confounding factors and also examined for effect modification by key covariates such as ApoE4, age and sex.

Conclusion and area for future research In this study, type 2 diabetes was associated with decline in verbal memory and fluency in older community-dwelling people without dementia over a period of $\sim 5$ years but not with MRI markers of brain atrophy. The effects of type 2 diabetes and poor metabolic health at midlife, and the impact of accrual of cerebrovascular lesions at older age, both deserve further study to inform preventative efforts against dementia.

Acknowledgements The results of this study have been presented in abstract form at the Alzheimer's Association International Conference, London, 2017, and the Australian Dementia Forum, Melbourne, 2017.

Data availability Data are available by request from the corresponding author.

Funding This study was funded by the National Health and Medical Research Council (NHMRC) (project grant 403000 and 436797), Australia. MLC is funded by an NHMRC Boosting Dementia Research Leadership Fellowship (1135761). CM is funded by an NHMRC/ARC Dementia Early Career Fellowship (1109482). VKS is a recipient of NHMRC project grants (403000 and 436797) and an NHMRC Practitioner Fellowship (APP1137837). WW, RB and TP have no funding to declare.

Duality of interest MLC, RB, CM, WW and VKS have no conflicts of interest to declare. TP is on the Genzyme advisory board on Fabry disease, and has received payment for lectures including service on speakers' bureaus for Bayer, Boehringer Ingelheim, Pfizer and Genzyme.
Contribution statement MLC drafted the manuscript and analysed the data. RB completed the image processing and analysis. MLC, $\mathrm{RB}, \mathrm{CM}, \mathrm{WW}, \mathrm{TP}$ and VKS interpreted the data and revised the manuscript. WW assisted with the statistical analysis. VKS was responsible for the study concept and design. All authors approved the final version of the manuscript. MLC and VKS are the guarantors of this work.

Publisher's Note Springer Nature remains neutral with regard to jurisdictional claims in published maps and institutional affiliations.

\section{References}

1. Alzheimer's Disease International (2014) World Alzheimer report. Alzheimer's Disease International, London

2. Cheng G, Huang C, Deng H, Wang H (2012) Diabetes as a risk factor for dementia and mild cognitive impairment: a meta-analysis of longitudinal studies. Intern Med J 42(5):484 491. https://doi.org/ 10.1111/j.1445-5994.2012.02758.x

3. Tuligenga RH, Dugravot A, Tabak AG et al (2014) Midlife type 2 diabetes and poor glycaemic control as risk factors for cognitive decline in early old age: a post-hoc analysis of the Whitehall II cohort study. Lancet Diabetes Endocrinol 2(3):228-235. https:// doi.org/10.1016/S2213-8587(13)70192-X

4. Spauwen PJ, Kohler S, Verhey FR, Stehouwer CD, van Boxtel MP (2013) Effects of type 2 diabetes on 12-year cognitive change: results from the Maastricht aging study. Diabetes Care 36(6): 1554-1561. https://doi.org/10.2337/dc12-0746

5. Rajan KB, Arvanitakis Z, Lynch EB et al (2016) Cognitive decline following incident and preexisting diabetes mellitus in a population sample. Neurology 87(16):1681-1687. https://doi.org/10.1212/ WNL.0000000000003226

6. Rawlings AM, Sharrett AR, Schneider AL et al (2014) Diabetes in midlife and cognitive change over 20 years: a cohort study. Ann Intern Med 161(11):785-793. https://doi.org/10.7326/M14-0737

7. Knopman DS, Mosley TH, Catellier DJ, Coker LH, Atherosclerosis Risk in Communities Study Brain MRI Study (2009) Fourteen-year longitudinal study of vascular risk factors, APOE genotype, and cognition: the ARIC MRI Study. Alzheimers Dement 5(3):207214. https://doi.org/10.1016/j.jalz.2009.01.027

8. Yaffe K, Falvey C, Hamilton N et al (2012) Diabetes, glucose control, and 9-year cognitive decline among older adults without dementia. Arch Neurol 69(9):1170-1175. https://doi.org/10.1001/ archneurol.2012.1117

9. Nooyens AC, Baan CA, Spijkerman AM, Verschuren WM (2010) Type 2 diabetes and cognitive decline in middle-aged men and women: the Doetinchem Cohort Study. Diabetes Care 33(9): 1964-1969. https://doi.org/10.2337/dc09-2038

10. Wisse LE, de Bresser J, Geerlings MI et al (2014) Global brain atrophy but not hippocampal atrophy is related to type 2 diabetes. $\mathrm{J}$ Neurol Sci 344(1-2):32-36. https://doi.org/10.1016/j.jns.2014.06.008

11. Moran C, Phan TG, Chen J et al (2013) Brain atrophy in type 2 diabetes: regional distribution and influence on cognition. Diabetes Care 36(12):4036-4042. https://doi.org/10.2337/dc13-0143

12. Biessels GJ, Reijmer YD (2014) Brain changes underlying cognitive dysfunction in diabetes: what can we learn from MRI? Diabetes 63(7):2244-2252. https://doi.org/10.2337/db14-0348

13. Roberts RO, Knopman DS, Przybelski SA et al (2014) Association of type 2 diabetes with brain atrophy and cognitive impairment. Neurology 82(13):1132-1141. https://doi.org/10.1212/WNL. 0000000000000269

14. Williamson JD, Launer LJ, Bryan RN et al (2014) Cognitive function and brain structure in persons with type 2 diabetes mellitus after intensive lowering of blood pressure and lipid levels: a randomized 
clinical trial. JAMA Intern Med 174(3):324-333. https://doi.org/10. 1001/jamainternmed.2013.13656

15. Launer LJ, Miller ME, Williamson JD et al (2011) Effects of intensive glucose lowering on brain structure and function in people with type 2 diabetes (ACCORD MIND): a randomised open-label substudy. Lancet Neurol 10(11):969-977. https://doi.org/10.1016/ S1474-4422(11)70188-0

16. Kooistra M, Geerlings MI, Mali WP et al (2013) Diabetes mellitus and progression of vascular brain lesions and brain atrophy in patients with symptomatic atherosclerotic disease. The SMART-MR study. J Neurol Sci 332(1-2):69-74. https://doi.org/10.1016/j.jns. 2013.06.019

17. de Bresser J, Tiehuis AM, van den Berg E et al (2010) Progression of cerebral atrophy and white matter hyperintensities in patients with type 2 diabetes. Diabetes Care 33(6):1309-1314. https://doi. org/10.2337/dc09-1923

18. Debette S, Seshadri S, Beiser A et al (2011) Midlife vascular risk factor exposure accelerates structural brain aging and cognitive decline. Neurology 77(5):461-468. https://doi.org/10.1212/WNL. 0b013e318227b227

19. van Elderen SG, de Roos A, de Craen AJ et al (2010) Progression of brain atrophy and cognitive decline in diabetes mellitus: a 3-year follow-up. Neurology 75(11):997-1002. https://doi.org/10.1212/ WNL.0b013e3181f25f06

20. Espeland MA, Bryan RN, Goveas JS et al (2013) Influence of type 2 diabetes on brain volumes and changes in brain volumes: results from the Women's Health Initiative magnetic resonance imaging studies. Diabetes Care 36(1):90-97. https://doi.org/10.2337/dc120555

21. Lezak M (1995) Neuropsychological assessment. Oxford University Press, New York

22. Spreen O, Strauss E (1998) A compendium of neuropsychological tests. Administration, norms, and commentary. Oxford University Press, New York

23. Weschler D (1997) Weschler Adult Intelligence Scale. Psychological Corporation, New York

24. Reuter M, Schmansky NJ, Rosas HD, Fischl B (2012) Withinsubject template estimation for unbiased longitudinal image analysis. Neuroimage 61(4):1402-1418. https://doi.org/10.1016/j. neuroimage.2012.02.084

25. Choi P, Ren M, Phan TG et al (2012) Silent infarcts and cerebral microbleeds modify the associations of white matter lesions with gait and postural stability: population-based study. Stroke 43(6): 1505-1510. https://doi.org/10.1161/STROKEAHA.111.647271

26. Lee KJ, Roberts G, Doyle LW, Anderson PJ, Carlin JB (2016) Multiple imputation for missing data in a longitudinal cohort study: a tutorial based on a detailed case study involving imputation of missing outcome data. Int J Soc Res Methodol 19(5):575-591. https://doi.org/10.1080/13645579.2015.1126486
27. Enders CK (2010) Applied missing data analysis. Guilford Press, New York

28. Muthén LK, Muthén BO (1998-2018) Mplus user's guide. Available from www.statmodel.com/ugexcerpts.shtml. Accessed 14 May 2018

29. Asparouhov T, Muthén BO (2010) Multiple imputation with Mplus. Available from www.statmodel.com/download/ Imputations7.pdf. Accessed 14 May 2018

30. Graham J (2012) Missing data. Springer, New York, https://doi.org/ 10.1007/978-1-4614-4018-5

31. Schafer JL, Olsen MK (1998) Multiple imputation for multivariate missing-data problems: a data analyst's perspective. Multivar Behav Res 33(4):545-571. https://doi.org/10.1207/ s15327906mbr3304_5

32. Rubin DB (1987) Multiple imputation for nonresponse in surveys. Wiley, New York. https://doi.org/10.1002/9780470316696

33. Tomlin A, Sinclair A (2016) The influence of cognition on selfmanagement of type 2 diabetes in older people. Psychol Res Behav Manag 9:7-20. https://doi.org/10.2147/PRBM.S36238

34. Arvanitakis Z, Wilson RS, Bienias JL, Evans DA, Bennett DA (2004) Diabetes mellitus and risk of Alzheimer disease and decline in cognitive function. Arch Neurol 61(5):661-666. https://doi.org/ 10.1001/archneur.61.5.661

35. Maggi S, Limongi F, Noale $\mathrm{M}$ et al (2009) Diabetes as a risk factor for cognitive decline in older patients. Dement Geriatr Cogn Disord 27(1):24-33. https://doi.org/10.1159/000183842

36. Mayeda ER, Haan MN, Yaffe K, Kanaya AM, Neuhaus J (2015) Does type 2 diabetes increase rate of cognitive decline in older Mexican Americans? Alzheimer Dis Assoc Disord 29(3):206212. https://doi.org/10.1097/WAD.0000000000000083

37. Pappas C, Andel R, Infurna FJ, Seetharaman S (2017) Glycated haemoglobin $\left(\mathrm{HbA}_{1 \mathrm{c}}\right)$, diabetes and trajectories of change in episodic memory performance. J Epidemiol Community Health 71(2): 115-120. https://doi.org/10.1136/jech-2016-207588

38. Mayeda ER, Haan MN, Neuhaus J et al (2014) Type 2 diabetes and cognitive decline over 14 years in middle-aged African Americans and whites: the ARIC Brain MRI Study. Neuroepidemiology 43(34):220-227. https://doi.org/10.1159/000366506

39. Okereke OI, Kang JH, Cook NR et al (2008) Type 2 diabetes mellitus and cognitive decline in two large cohorts of communitydwelling older adults. J Am Geriatr Soc 56(6):1028-1036. https:// doi.org/10.1111/j.1532-5415.2008.01686.x

40. Bangen KJ, Gu Y, Gross AL et al (2015) Relationship between type 2 diabetes mellitus and cognitive change in a multiethnic elderly cohort. J Am Geriatr Soc 63(6):1075-1083. https://doi.org/10.1111/ jgs. 13441

41. van den Berg E, Reijmer YD, de Bresser J et al (2010) A 4 year follow-up study of cognitive functioning in patients with type 2 diabetes mellitus. Diabetologia 53(1):58-65. https://doi.org/10. 1007/s00125-009-1571-9 